\title{
Facile Construction of Near Infrared Fluorescence Nanoprobe with Amphiphilic Protein-Polymer Bioconjugate for Targeted Cell Imaging
}

\author{
Zhongyun Liu, ${ }^{\dagger,}, \perp \mathrm{Na}$ Chen, ${ }^{\S, \perp}$ Chunhong Dong, ${ }^{\dagger}$ Wei Li, ${ }^{\|}$Weisheng Guo, ${ }^{\dagger}$ Hanjie Wang, ${ }^{\dagger}$ \\ Sheng Wang, Jian Tan, "Yu Tu, ${ }^{\dagger}$, and Jin Chang ${ }^{*}, \dagger$ \\ ${ }^{\dagger}$ School of Life Sciences, Tianjin University and Tianjin Engineering Center of Micro-Nano Biomaterials and Detection-Treatment \\ Technology, Tianjin 300072, P.R. China \\ ${ }^{\ddagger}$ Yantai Institute of Coastal Zone Research, Chinese Academy of Sciences, Yantai, Shandong 264003, P.R. China \\ ${ }^{\S}$ Department of Medical Radioprotection, School of Radiation Medicine and Health, Jiangsu Provincial Key Laboratory of Radiation \\ Medicine and Protection, Soochow University, Suzhou, 200072, P. R. China \\ "Department of Nuclear Medicine, Tianjin Medical University General Hospital, Tianjin 300052, P. R. China
}

\section{Supporting Information}

\begin{abstract}
A simple, straightforward, and reproducible strategy for the construction of a near-infrared (NIR) fluorescence nanoprobe was developed by coating $\mathrm{CuInS}_{2} / \mathrm{ZnS}$ quantum dots (CIS/ZnS QDs) with a novel amphiphilic bioconjugate. The amphiphilic bioconjugate with a tailor-designed structure of bovine serum albumin (BSA) as the hydrophilic segment and poly ( $\varepsilon$-caprolactone) (PCL) as the hydrophobic part was fabricated by chemical coupling the hydrophobic polymer chain to BSA via the maleimide-sulfhydryl reaction. By incorporating CIS/ZnS QDs into the hydrophobic cores of the self-assembly of BSA-PCL conjugate, the constructed NIR

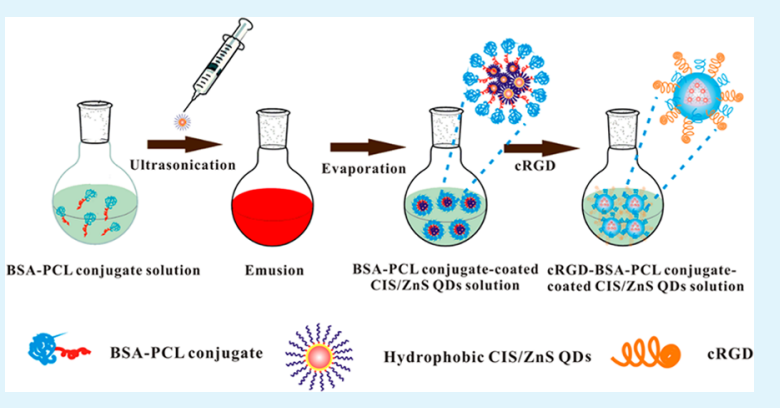
fluorescence nanoprobe exhibited excellent fluorescent properties over a wide $\mathrm{pH}$ range $(\mathrm{pH} 3-10)$ and a good colloidal stability in PBS buffer $(\mathrm{pH}=7.4)$ with or without $10 \%$ fetal bovine serum. The presence of the outer BSA shell effectively reduced the nonspecific cellular binding and imparted high biocompatibility and low-toxicity to the probe. Moreover, the NIR fluorescence nanoprobe could be functionalized by conjugating cyclic Arg-Gly-Asp (cRGD) peptide, and the decorated nanoprobe was shown to be highly selective for targeted integrin $\alpha_{\mathrm{v}} \beta_{3}$-overexpressed tumor cell imaging. The feasibility of the constructed NIR fluorescence probe in vivo application was further investigated and the results demonstrated its great potential for in vivo imaging. This developed protocol for phase transfer of the CIS/ZnS QDs was universal and applicable to other nanoparticles stabilized with hydrophobic ligands.
\end{abstract}

KEYWORDS: protein-polymer conjugate, BSA, self-assembly, CuIn $\mathrm{S}_{2} / \mathrm{ZnS}$ quantum dots, fluorescence nanoprobe

\section{INTRODUCTION}

Recently, nanoparticle-based near-infrared (NIR) fluorescence imaging has drawn much attention in noninvasive biological imaging due to its high sensitivity, high spatial resolution, deep tissue penetration, and less damage to biological samples. ${ }^{1-6}$ In particular, $\mathrm{CuInS}_{2} / \mathrm{ZnS}$ quantum dots (CIS/ZnS QDs) have been considered to be potential candidates as they are not only cadmium-free but also provide high extinction coefficients and photoluminescence (PL) emission ranging from the visible to the NIR. ${ }^{7-10}$ The cadmium-free element composition imparts CIS/ZnS QDs low toxicity and NIR emission leads to relatively deep tissues penetration. Despite the great advantages in the composition and the optical properties of CIS/ZnS QDs, some critical issues still remain to be addressed to construct the optimal fluorescence probes with CIS/ZnS QDs for in vitro and in vivo imaging. For example, the performed CIS/ZnS QDs via the classical organic-phase method are capped with hydrophobic alkyl ligands and are not soluble in aqueous solution, which significantly hampers their applications in biomedical fields. ${ }^{11}$ In addition, how to reduce the nonspecific binding and enhance the targeted cell imaging is another challenge for CIS/ $\mathrm{ZnS}$ QDs to be applied to in vitro and in vivo imaging. ${ }^{12,13}$ Therefore, rational surface engineering and functionalization of CIS/ZnS QDs are of great significance. ${ }^{14,15}$

Until now, various strategies for surface modification of QDs have been developed. Among them, coating of QDs with amphiphilic polymers via hydrophobic interactions is considered to be a promising, relatively easy, and robust approach. $^{16-18}$ This approach does not perturb the original hydrophobic surface ligands of the QDs and leads to high photoluminescence after modification. However, amphiphilic polymers used to modify the CIS/ZnS QDs surface have been rarely reported, and the polymers reveal one or more limitations in their biocompatibility and their capacity in

Received: April 2, 2015

Accepted: August 11, 2015

Published: August 11, 2015 
reducing nonspecific cellular binding. Bovine serum albumin (BSA), as a nonspecific binding blocking agent, is frequently used for engineering the surface of QDs, and it has been demonstrated that the BSA-coated QDs can effectively reduce the nonspecific cellular binding due to increasing the electrostatic repulsion and decreasing the hydrophobic interactions between QDs and cell membranes. ${ }^{19-22}$ However, the reported BSA-coated CIS/ZnS QDs are prepared either through a relatively complicated process of covalent binding between BSA and the surface of QDs or replacing the original ligands of QDs via ligand exchange with loss of fluorescence. Therefore, there is still a high need to develop innovative materials for constructing NIR fluorescence probes with excellent fluorescent properties, good biocompatibility, and low nonspecific cellular binding simultaneously.

Herein, we present a simple, straightforward, and reproducible strategy for constructing a NIR fluorescence probe via surface engineering of CIS/ZnS QDs with a novel amphiphilic bioconjugate. This amphiphilic bioconjugate with a tailordesigned structure of BSA as the hydrophilic segment and poly ( $\varepsilon$-caprolactone) (PCL) as the hydrophobic part was fabricated by chemical coupling the PCL chain to BSA via the maleimide-sulfhydryl reaction. This developed BSA-PCL conjugate displayed excellent biodegradability, good biocompatibility, and similar self-assembly behaviors to the traditional amphiphilic block copolymers and had been shown to selfassemble into nanosized micelles in the aqueous phase. ${ }^{23}$ The NIR fluorescence probe was constructed via encapsulating the CIS/ZnS QDs into the core of the self-assembly of the BSAPCL bioconjugate without perturbing the original hydrophobic surface ligands of the CIS/ZnS QDs, and consequently the probe exhibited excellent fluorescence properties and good colloidal stability. The presence of the BSA shell located in the outer layer could effectively reduce the nonspecific cellular binding of the probe and render high biocompatibility and lowtoxicity to the probe. This strategy merged the advantages of the modification methods with amphiphilic polymers and BSA. Further, targeted ligand, cyclic Arg-Gly-Asp (cRGD) peptide, was conjugated to the developed probe by utilizing the functional groups in the BSA, and the decorated probe was highly selective for targeted integrin $\alpha_{\mathrm{v}} \beta_{3}$-expressed cell imaging.

\section{EXPERIMENTAL SECTION}

2.1. Materials. $\mathrm{CuInS}_{2} / \mathrm{ZnS}$ quantum dots were synthesized as previously described. ${ }^{24}$ Dithiothreitol (DTT) was purchased from Sigma-Aldrich (St. Louis, MO). BSA (lyophilized powder, A3294) was purchased from Sigma-Aldrich and used after further reduced treatment via DTT. $N$-(2-Hydroxyethyl) maleimide was synthesized as previously described, ${ }^{23}$ and $\varepsilon$-caprolactone was obtained from Aldrich and used without further purification. $N$-(3-(Dimethylamino)propyl)- $N$-ethylcarbodiimide hydrochloride (EDC) and N-hydroxysuccinimide (NHS) were obtained from GL Biochem (Shanghai) Ltd. Arg-Gly-Asp peptide (c(RGDfK)) was obtained from Dingguo Biotechnology Co. Ltd. (Beijing, China). All other chemicals were obtained from local suppliers and used as received.

2.2. Preparation of Amphiphilic BSA-PCL Conjugate. The coupling of the maleimide-functionalized PCL to the reduced BSA was performed in a DMF/water mixture $(10 / 90, \mathrm{v} / \mathrm{v})$ according to our previous reported method. ${ }^{23}$ Briefly, maleimide-functionalized PCL(Mal-PCL) was first synthesized by ring-opening polymerization (ROP) of $\varepsilon$-caprolactone in the presence of $\mathrm{N}$-(2-hydroxyethyl)maleimide as an initiator and stannous octoate as a catalyst, and the molecular weight of it, as determined by GPC, was $3216 \mathrm{Da}$. The DMF solution of Mal-PCL $(0.15 \mathrm{mM})$ was added to the reduced BSA in water $(0.015 \mathrm{mM}, 100 \mathrm{mM}$ phosphate buffer, $\mathrm{pH}=6.8,2 \mathrm{mM}$ EDTA, $150 \mathrm{mM} \mathrm{NaCl})$. The reaction was allowed to proceed overnight, and the excess of reactants was removed by extensive dialysis against DMF and pure water for $48 \mathrm{~h}$ by a dialysis bag $(\mathrm{MWCO}=35000 \mathrm{Da})$. The powder of BSA-PCL conjugate was obtained by the freeze-drying procedure and was further purified via a Superdex 150 column eluting with $20 \mathrm{mM}$ phosphate buffer $(\mathrm{pH}=$ 7.4).

2.3. Surface Modification of CIS/ZnS QDs with Amphiphilic BSA-PCL Conjugate. The hydrophobic dodecanethiol-capped CIS/ ZnS QDs were prepared in our group. ${ }^{24}$ In this study, the prepared CIS/ZnS QDs were transferred into the aqueous phase successfully via encapsulating the CIS/ZnS QDs with BSA-PCL conjugate. In brief, 16 $\mathrm{mg}$ of BSA-PCL conjugates were dissolved in $4 \mathrm{~mL}$ of deionized water at room temperature and sonicated in a bath sonication for $10 \mathrm{~min}$. During the ultrasonic treatment process, the $2 \mathrm{~mL}$ of preformed dichloromethane solutions containing CIS/ZnS QDs were slowly injected into the deionized water via a syringe. Dichloromethane was then evaporated with a vacuum rotary evaporator and finally, the QDs dispersed in aqueous phase were purified by centrifugation at $20000 \mathrm{~g}$ for $30 \mathrm{~min}$ and washed with deionized water three times to remove residual self-assembly of BSA-PCL. The constructed NIR fluorescence nanoprobe was kept at $4{ }^{\circ} \mathrm{C}$ until further characterization was done.

2.4. Conjugation of the NIR Fluorescence Nanoprobe with cRGD Peptide. The NIR fluorescence nanoprobe decorated with targeting ligand was prepared via coupling cRGD peptide to the surface of the developed QDs utilizing carbodiimine chemistry. Typically, the NIR fluorescence nanoprobe solution was activated with $\mathrm{EDC} \cdot \mathrm{HCl}$ and $\mathrm{NHS}$ for $15 \mathrm{~min}$ at room temperature with a rolling incubator. Then cRGD peptide was added into the activated QDs solution and the mixture was continuously stirred for $3 \mathrm{~h}$. The mixture solution was centrifuged at $20000 \mathrm{rpm}$ for $30 \mathrm{~min}$ and washing with deionized water three times, and the purified QDs solution was stored at $4{ }^{\circ} \mathrm{C}$ for use.

2.5. Cellular Uptake and Targeting Image. U87 and HeLa cells were seeded into a small plate at a density of $1.0 \times 10^{5}$ cells per well and incubated in $3 \mathrm{~mL}$ of DMEM containing $10 \% \mathrm{FBS}$ at $37^{\circ} \mathrm{C}$ in the incubator for $24 \mathrm{~h}$. Then $100.0 \mu \mathrm{L}$ of NIR fluorescence nanoprobe solutions and cRGD-decorated NIR fluorescence nanoprobe solutions were added to the wells. After the cells had been incubated for $2 \mathrm{~h}$, the cells were washed three times with PBS buffer and then fixed with $4 \%$ paraformaldehyde for $15 \mathrm{~min}$. Finally, the fixed cells were incubated with $300 \mu \mathrm{L}$ of DAPI solution $(10 \mu \mathrm{g} / \mathrm{mL})$ for $15 \mathrm{~min}$ at $37{ }^{\circ} \mathrm{C}$ for nucleus staining. The cells were washed three times with PBS buffer and observed by confocal laser scanning microscopy (CLSM) (Leica MicrosystemsGmbH, TCS SP 2).

Cellular binding and uptake of BSA-PCL conjugate coated CIS/ZnS QDs and cRGD-BSA-PCL conjugate coated CIS/ZnS QDs were further evaluated by flow cytometry. U87 and HeLa cells were seeded into a small plate at a density of $1.0 \times 10^{5}$ cells per well and cultured with Dulbecco's modified Eagle's medium (DMEM, containing 10\% fetal bovine serum, $1 \%$ penicillin, and $1 \%$ streptomycin) in the incubator under a $5 \% \mathrm{CO}_{2}$ atmosphere at $37^{\circ} \mathrm{C}$ for $24 \mathrm{~h}$. Then, 100.0 $\mu \mathrm{L}$ of BSA-PCL conjugate coated CIS/ZnS QDs solutions and cRGDBSA-PCL conjugate coated CIS/ZnS QDs solutions were added to the wells. After incubation for $2 \mathrm{~h}$, cells were washed three times with PBS buffer and harvested. The analysis was examined by a flow cytometer on an FACSCalibur (BD Biosciences).

2.6. In Vitro Cytotoxicity. The MTT assay was used to assess the cytotoxicity of the blank micelle formed by the self-assembly of the BSA-PCL conjugate and the formed BSA-PCL conjugate coated CIS/ ZnS QDs. In brief, U87 and HeLa cells were seeded in 96-well microplates at a density of $5 \times 10^{3}$ cells/well and cultured with Dulbecco's modified Eagle's medium (DMEM, containing 10\% fetal bovine serum, $1 \%$ penicillin, and $1 \%$ streptomycin) in the incubator under a $5 \% \mathrm{CO}_{2}$ atmosphere at $37{ }^{\circ} \mathrm{C}$ for $24 \mathrm{~h}$. Then, $100.0 \mu \mathrm{L}$ of medium containing different blank micelle solutions and BSA-PCL conjugate coated CIS/ZnS QDs solutions were added to the wells, and $100 \mu \mathrm{L}$ of medium was added to the wells containing control cells. The cells were further incubated for $24 \mathrm{~h}$, and the MTT solution (5 mg/ 


\section{Amphiphilic Protein-Polymer Conjugate}

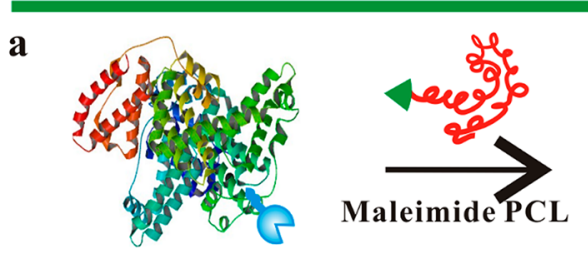

BSA

b
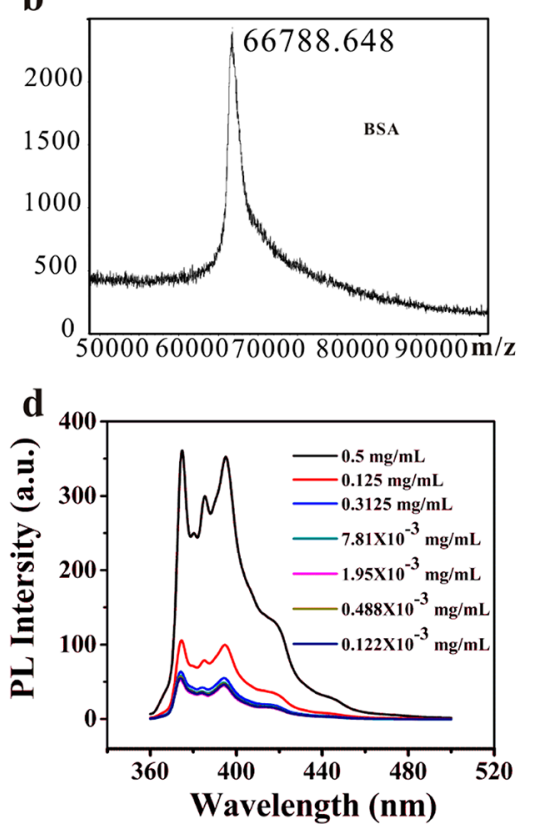

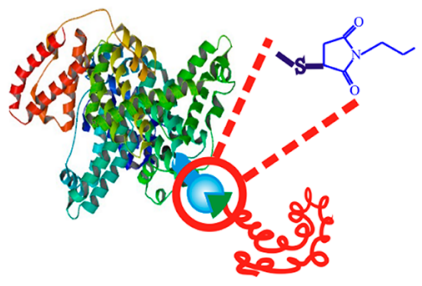

BSA-PCL Conjugate
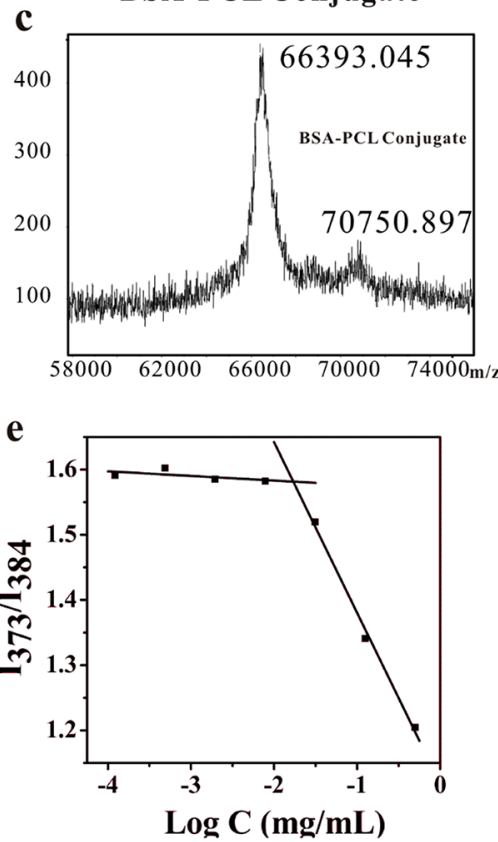

Figure 1. (a) Schematic illustration of the preparation process of amphiphilic BSA-PCL conjugate, (b) MALDI-TOF spectra of native BSA conjugate, (c) MALDI-TOF spectra of the BSA-PCL conjugate, (d) fluorescence emission spectra of pyrene measured at various concentrations of BSA-PCL conjugate, (e) ratio of fluorescence intensities at 373 and $384 \mathrm{~nm}\left(I_{373} / I_{384}\right)$ plotted against the logarithm concentrations of the amphiphilic BSA-PCL conjugate.

$\mathrm{mL}$ in water) was added to the wells $(20 \mu \mathrm{L} /$ well $)$. After being incubated at $37^{\circ} \mathrm{C}$ for $4 \mathrm{~h}$, the medium was replaced with $200 \mu \mathrm{L}$ of DMSO. The 96-well microplate was shaken with a shaking table for 10 min to dissolve the blue formazan precipitates, and the UV absorption of formazan at $490 \mathrm{~nm}$ was measured using a microplate reader. The cell survival rate was then calculated from the microplate reader of cells relative to that of nontreated cells.

2.7. In Vivo Fluorescence Imaging. All animal experiments were performed in compliance with the UK Home Office Code of Practice for the Housing and Care of Animals Used in Scientific Procedures. Five-week-old normal female CD-1 nude mice (Charles River Laboratories, U.K.) were used in this study. The mice were feed only with water for $12 \mathrm{~h}$ before injection of QDs to minimize food fluorescence. Then $200 \mu \mathrm{L}$ of BSA-PCL conjugate coated CIS/ZnS QDs solution containing $100 \mu \mathrm{g}$ of CIS/ZnS QDs were injected intravenously via the tail vein, and subsequently whole body images were performed with the Maestro In-Vivo Imaging System and analyzed at various time points.

2.8. Characterization. ${ }^{1} \mathrm{H}$ NMR spectra of PCL were obtained using a Varian Inova-500 MHz instrument (Varian Inc., Palo Alto, CA) with $\mathrm{CDCl}_{3}$ as the solvent and TMS as an internal standard. The average molecular weight of PCL and the polydispersity index (PDI) of the polymer were confirmed using a Waters 1515 gel permeation chromatograph (GPC, Waters Co.), and polystyrene with a narrow molecular weight distribution was the calibration standard. The elution phase was THF at a flow rate of $1.0 \mathrm{~mL} / \mathrm{min}$. Matrix-assisted laser desorption ionization time-of-flight mass spectroscopy (MALDI-TOF
MS, Autoflex TOF/TOFIII, Bruker Daltonics Inc.) and sodium dodecyl sulfate-polyacrylamide gel electrophoresis (SDS-PAGE) were employed to determine the molecular weights of BSA and the BSAPCL conjugate. The PL spectra of QDs before and after the modification were acquired by using a Gangdong F-280 spectrofluorometer, and the quantum yields (QYs) were estimated by comparison of the integrated fluorescence intensity with standard Rhodamin 6G (95\% in ethanol) solutions with the same optical density $(0.05-0.1)$ at the excitation wavelength $(470 \mathrm{~nm})$. Transmission electron microscopy (TEM) images were performed on a JEOL-100CXII instrument in bright-field mode with an operating voltage of $100 \mathrm{kV}$, and the high-resolution TEM images were taken on Tecnai G2 F20 instrument operated at $200 \mathrm{kV}$. The solutions of selfassembled BSA-PCL conjugates $(2.0 \mathrm{mg} / \mathrm{mL})$ were dropped onto a carbon-coated copper grid and allowed to dry in air at room temperature, and the hydrophobic QDs sample was dropped onto a carbon-coated nickel grid in the hexanes and dried at ambient temperature. The size, PDI, and surface charge of the self-assembly of BSA-PCL conjugate, BSA-PCL conjugate coated CIS/ZnS QDs, and cRGD-decorated BSA-PCL conjugate coated CIS/ZnS QDs were determined by DLS using a Brookhaven Zetasizer (Brookhaven Instruments Ltd.) at $25{ }^{\circ} \mathrm{C}$.

\section{RESULTS AND DISCUSSION}

3.1. Preparation of Amphiphilic BSA-PCL Conjugate and the Construction of the NIR Fluorescence Nanoprobe. The schematic illustration of the preparation process of 

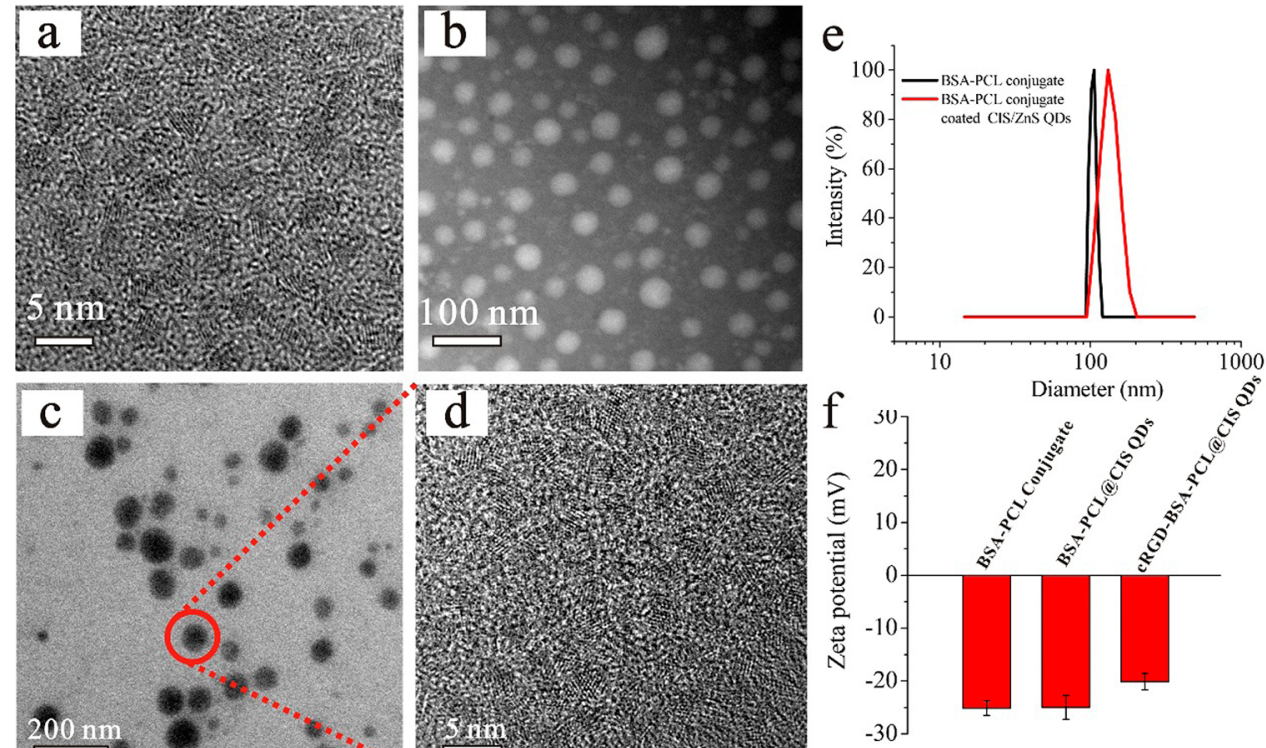

Figure 2. (a) TEM images of CIS/ZnS QDs dispersed in dichloromethane, (b) the blank self-assembly of bioblock BSA-PCL conjugate, (c) the NIR fluorescence nanoprobe dispersed in aqueous phase, (d) magnified high-resolution TEM image of the NIR fluorescence nanoprobe, (e) size and size distribution of the blank self-assembly of bioblock BSA-PCL conjugate and the NIR fluorescence nanoprobe in aqueous phase measured by DLS, (f) zeta potential of the blank self-assembly of BSA-PCL conjugate, the NIR fluorescence nanoprobe, and the cRGD-decorated NIR fluorescence nanoprobe.

Scheme 1. Construction of the NIR Fluorescence Nanoprobe with Amphiphilic BSA-PCL Conjugate and CIS/ZnS QDs

\section{The protocol for phase transfer of hydrophobic CIS/ZnS QDs}

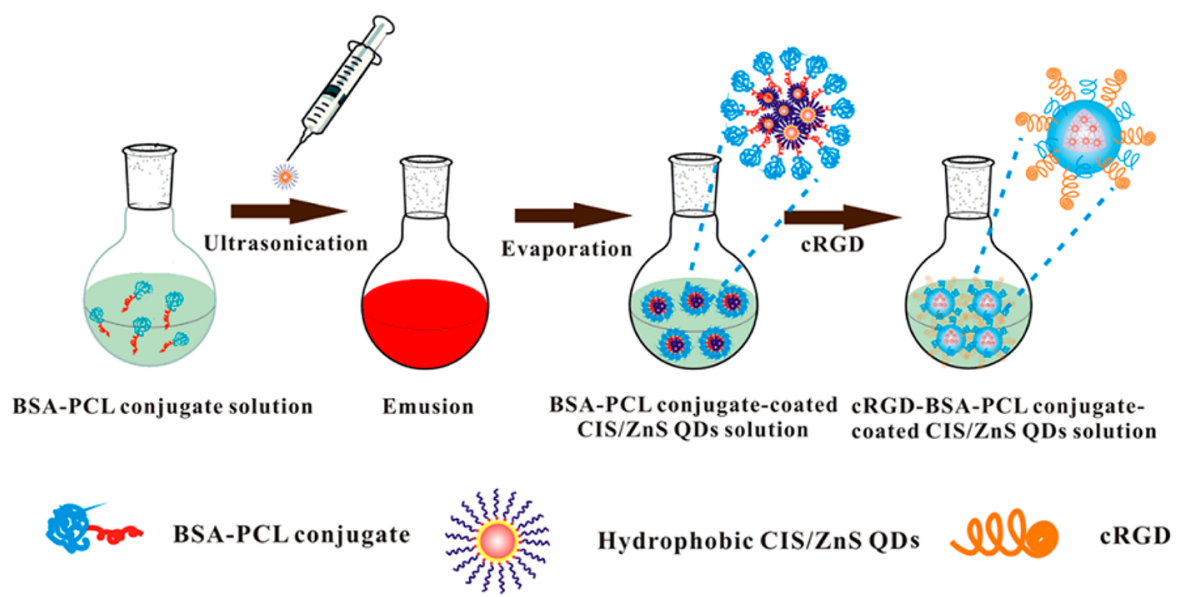

amphiphilic BSA-PCL conjugate was shown in Figure 1a. Amphiphilic protein-polymer conjugates represent promising candidates for efficient drug and imaging agent delivery due to meeting requirements such as low cytotoxicity, low immunogenicity, good biocompatibility, and ability to functionalize. $^{25-30}$ In this study, a hydrophobic Mal-PCL polymer was synthesized and employed to covalently link to BSA to prepare the BSA-PCL conjugate (the chemical structure and the molecular weight of the Mal-PCL is demonstrated in Figures S1 and S2 and Table S1 of the Supporting Information) and the molecular weights of the obtained BSA-PCL conjugate and native BSA were measured by MALDI-TOF MS and SDSPAGE. As shown in Figure 1b,c, the molecular weights of native BSA and BSA-PCL conjugate were determined to be 66788 and $70750 \mathrm{Da}$, respectively. This demonstrated that the synthetic PCL was successfully conjugated to BSA. The CMC of the performed amphiphilic BSA-PCL conjugate was determined as about $17.43 \mathrm{mg} / \mathrm{L}$ using pyrene as a hydrophobic probe (Figure 1d,e) and the obtained BSA-PCL conjugate could self-assemble into a nanosized micelle in the aqueous phase (Figure $2 \mathrm{~b}$ ).

Hydrophobic CIS/ZnS QDs capped with dodecanethiol (DDT) were prepared following our previous protocols, ${ }^{24}$ and the TEM image of the QDs was shown in Figure 2a. From the image, it could be seen that the synthesized QDs displayed clear lattice planes and good crystallinity with diameters of $\sim 3$ $\mathrm{nm}$. The phase transfer of CIS/ZnS QDs was achieved successfully via encapsulating the QDs into the core of the 

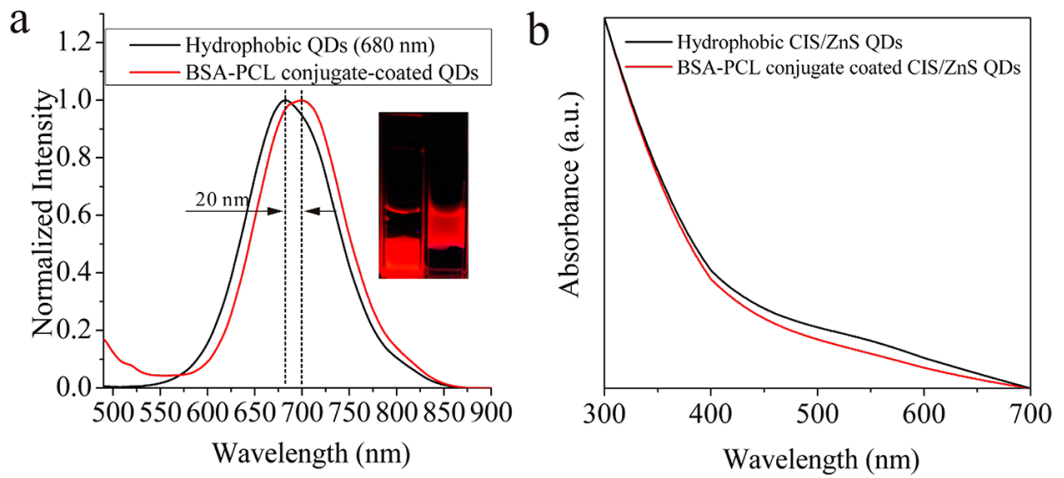

Figure 3. (a) Fluorescence emission spectra of as-prepared CIS/ZnS QDs (black) and BSA-PCL conjugate-coated CIS/ZnS QDs (red) with excitation wavelength at $470 \mathrm{~nm}$ and insert in part a showed the corresponding fluorescence image in the dark field and (b) UV-vis absorption spectrum of original CIS/ZnS QDs (black) and BSA-PCL conjugate-coated QDs (red).
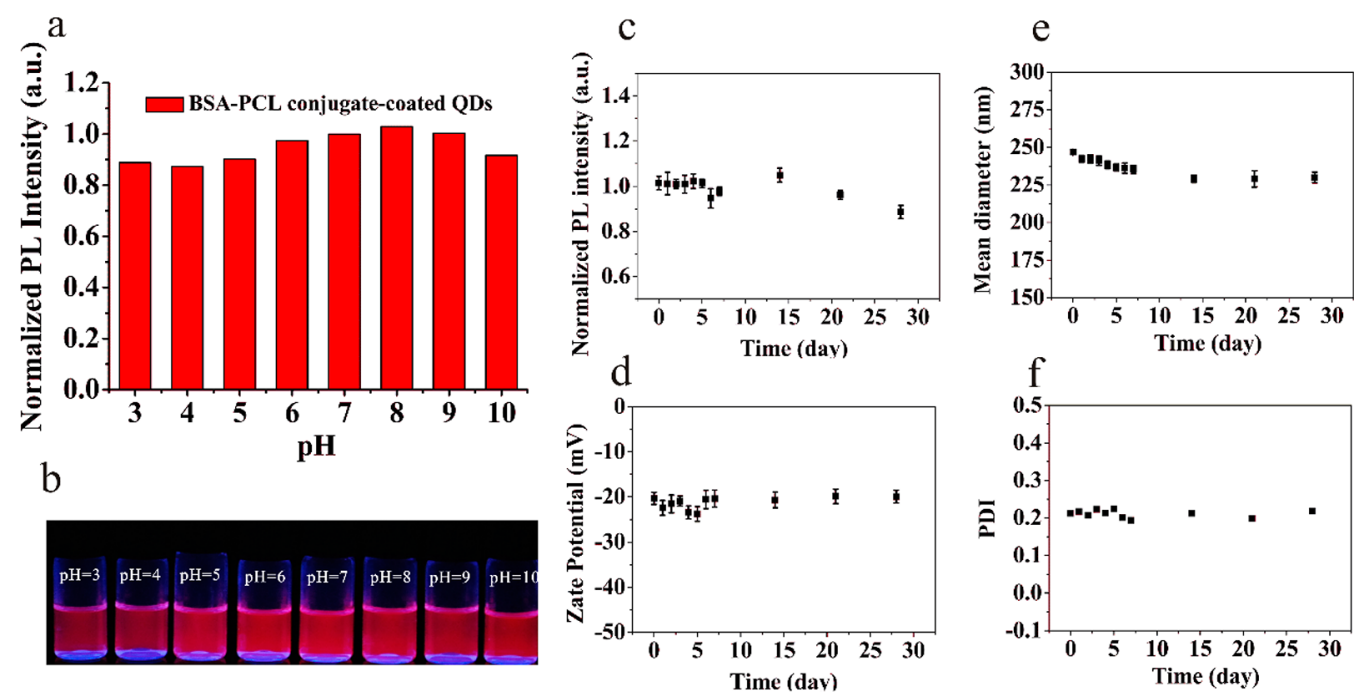

Figure 4. (a) Fluorescence intensities and (b) corresponding fluorescence image in the dark field of the NIR fluorescence nanoprobe in different $\mathrm{pH}$ value conditions; the fluorescence intensities (c), mean diameters (d), zeta potentials (e), and PDI (f) of the NIR fluorescence nanoprobe in PBS buffer $(\mathrm{pH}=7.4)$ with $10 \% \mathrm{FBS}$ at $37^{\circ} \mathrm{C}$ at different times.

micelle which was formed by the self-assembly of the performed BSA-PCL bioconjugate. The protocol for phase transfer was shown in Scheme 1. In brief, during the ultrasonic treatment process, dichloromethane solutions containing CIS/ $\mathrm{ZnS}$ QDs were slowly injected via syringe into the BSA-PCL conjugate solution with $4 \mathrm{mg} / \mathrm{mL}$. Upon injection, organic solvent was evaporated with a vacuum rotary evaporator and the BSA-PCL conjugate coated CIS/ZnS QDs solution was obtained. Finally, the QDs dispersed in the aqueous phase were purified by centrifugation at $20000 \mathrm{~g}$ for $30 \mathrm{~min}$ and washing with deionized water three times to remove residual from the blank self-assembly of the BSA-PCL conjugate. Figure 2b,c showed the morphologies of the micelle formed from the selfassembly of BSA-PCL conjugate without CIS/ZnS QDs and the BSA-PCL conjugate coated CIS/ZnS QDs nanoprobe, respectively. Also it was observed that there was an obvious contrast change after the incorporation of the CIS/ZnS QDs. The magnified high-resolution TEM image further verified the incorporation of the CIS/ZnS QDs and showed multiple QDs were encapsulated into each micelle (Figure 2d). The hydrodynamic size and size distribution of the blank micelle and the BSA-PCL conjugate coated CIS/ZnS QDs nanoprobe were determined by DLS in an aqueous environment (Figure 2e). The DLS results showed a hydrodynamic diameter of
$128.9 \pm 1.4 \mathrm{~nm}$ for BSA-PCL conjugate coated CIS/ZnS QDs (dispersity index of 0.132 ). The diameter determined by TEM observations was smaller than those from DLS measurement, which was because the BSA-PCL conjugate coatings shrinked substantially upon drying. ${ }^{31}$ Besides, the surface charges of the obtained blank self-assemble of bioblock BSA-PCL conjugate, the NIR fluorescence nanoprobe and the cRGD-decorated NIR fluorescence nanoprobe were measured. As shown in Figure 2f, the zeta potential values of the blank self-assembly of BSA-PCL conjugate and BSA-PCL@CIS QDs nanoprobe were -25.11 \pm $1.37 \mathrm{mV}$ and $-24.98 \pm 2.25 \mathrm{mV}$, respectively. Also, only a little change occurred when the BSA-PCL@CIS QDs nanoprobe was further decorated with cRGD, which was probably due to the introduction of cRGD on the surface of QDs. The cRGDdecorated QDs nanoprobe had a zeta potential of about -20.12 $\pm 1.56 \mathrm{mV}$. The mechanism of encapsulation for CIS/ZnS QDs with BSA-PCL conjugate was considered to be hydrophobic interactions between the surface ligands of the CIS/ZnS QDs and the hydrophobic parts of the micelle. To further verify the mechanism of the phase transfer, oleic acid (OA)-capped hydrophobic $\mathrm{Fe}_{3} \mathrm{O}_{4}$ magnetic nanoparticles were used to be modified with the BSA-PCL conjugate in the same way and quite similar phase transfer behaviors were found with the same modification approach (Figure S3 of the Supporting 


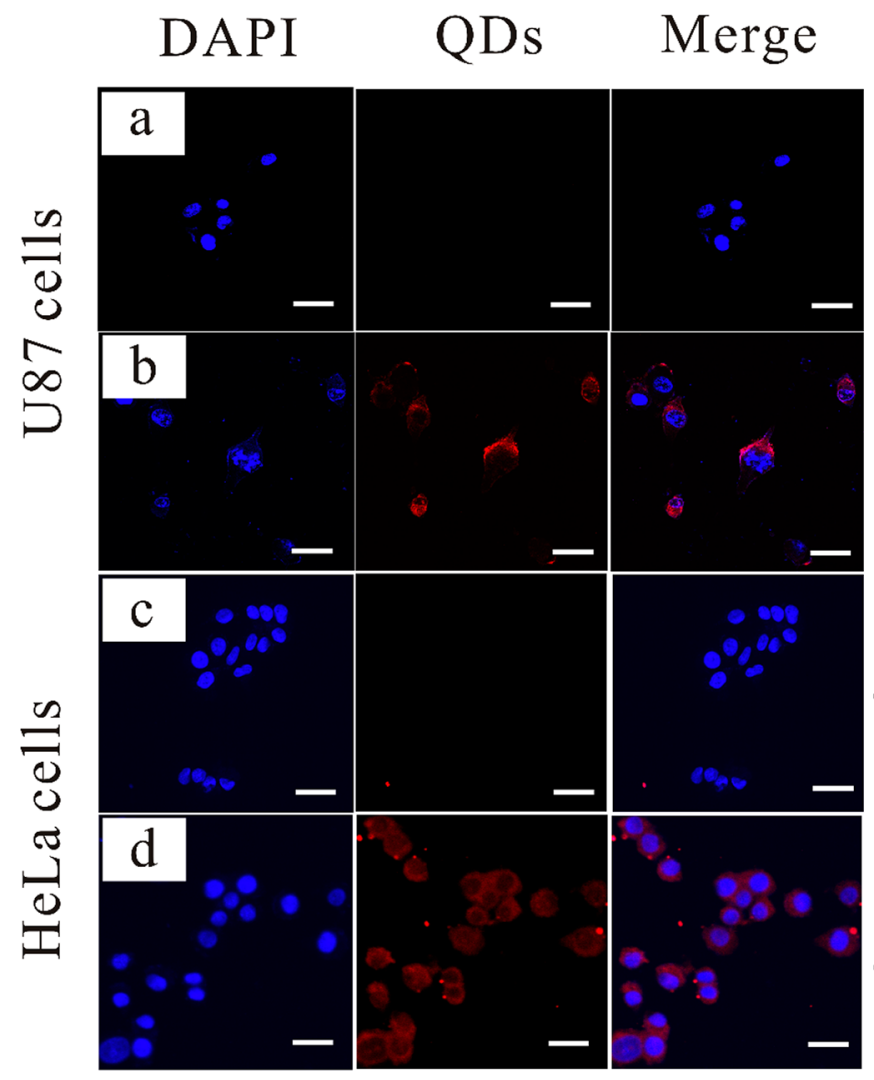

Flow cytometry

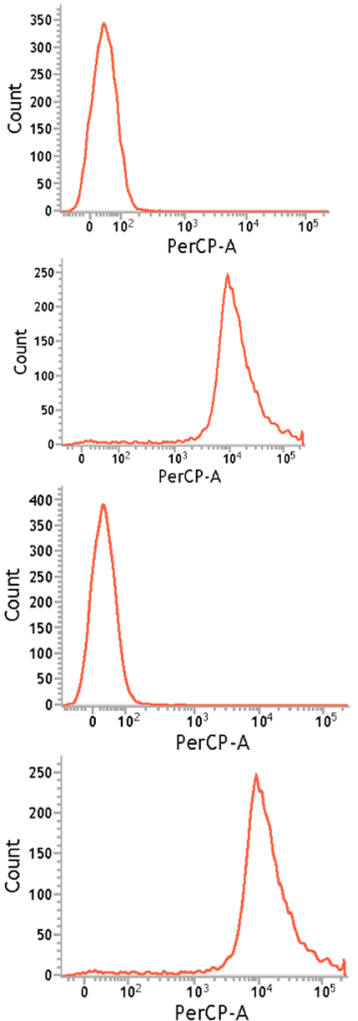

Figure 5. Confocal fluorescence image and fluorescence intensity of (a) U87 cells treated with nontargeting nanoprobe, (b) U87 cells treated with cRGD-functionalized nanoprobe, (c) HeLa cells treated with nontargeting nanoprobe, and (d) HeLa cells treated with cRGD-functionalized nanoprobe measured by CLSM and flow cytometry, respectively. The scale bars were $20 \mu \mathrm{m}$.

Information), demonstrating that this phase transfer method utilizing the amphiphilic BSA-PCL conjugate was universal and applicable to other nanoparticle stabilized with hydrophobic ligands.

3.2. Fluorescence Performance and Stability of the Constructed NIR Fluorescence Nanoprobe. The excellent fluorescence performances of fluorescent nanoprobes are crucially important for their applications. ${ }^{32}$ In this study, CIS/ZnS QDs with emission wavelength in $680 \mathrm{~nm}$ was used to construct NIR-emitted nanoprobe with the tailor-designed amphiphilic BSA-PCL conjugate. The fluorescence emission spectra and UV absorption spectra of CIS/ZnS QDs before and after the phase transfer were shown in Figure 3. The emission wavelength of CIS/ZnS QDs coated with BSA-PCL conjugate in the aqueous phase was $705 \mathrm{~nm}$ and revealed a slight bathochromic shift relative to the as-synthesized CIS/ZnS QDs in dichloromethane, and the reason may be multiple encapsulation of QDs into one nanoparticle. The quantum yield (QY) of the constructed nanoprobe decreased slightly from $\sim 13.87 \%$ in dichloromethane to $\sim 10.37 \%$ on average in water.

The fluorescence stability of the nanoprobe is of particular importance for in vitro and in vivo imaging. ${ }^{33,34}$ It was demonstrated that there was a prominent $\mathrm{pH}$ shift during cellular uptake of the fluorescence nanoprobe from blood or extracellular spaces $(\mathrm{pH} 7.2-7.4)$ to the various intracellular compartments ( $\mathrm{pH} 4.0-6.5),{ }^{35-37}$ and the fluorescence might decay drastically upon the condition of a low $\mathrm{pH}$ value. Considering the various $\mathrm{pH}$ gradient in the living body, the fluorescence stability of the BSA-PCL conjugate coated CIS/
ZnS QDs was tested over a wide $\mathrm{pH}$ range ( $\mathrm{pH} 3-10)$, and the results were shown in Figure 4a,b. According to the results, there was no obvious change in the fluorescence intensity, which was attributed to synergy between the retention of the original ligands of the QDs and the tightly packed BSA-PCL conjugate on the surface of the QDs. In addition, we found that the constructed nanoprobe was stable and the fluorescence intensities, the hydrodynamic diameters, zeta potentials, and polydispersity indexes (PDI) of the NIR fluorescence nanoprobe were little or not changed by the monitoring of the nanoprobe stored in PBS buffer with or without $10 \%$ fetal bovine serum (FBS) at $37{ }^{\circ} \mathrm{C}$ for 4 weeks, as shown in Figure $\mathrm{S} 4$ and Figure $4 \mathrm{c}-\mathrm{f}$. The excellent colloidal stability in PBS buffer with or without $10 \%$ FBS benefited from the protection of BSA which was on the outer surface of the nanoprobe, and the BSA shell could effectively reduce protein adsorption and consequently prevented the aggregation of the nanoprobe.

3.3. Targeted Cellular Imaging of the NIR Fluorescence Nanoprobe. BSA, as a nonspecific binding blocking agent, is frequently used for engineering the surface of QDs in biological detection and imaging. It has been demonstrated that the BSA-coated QDs could effectively reduce the nonspecific cellular binding due to increasing the electrostatic repulsion between QDs and cell membranes and decreasing the hydrophobic interactions between the ligands of QDs and lipids on the cell membranes, consequently reducing the nonspecific cellular imaging. In our study, we incubated the BSA-PCL conjugate coated nanoprobe with U87 and HeLa cells and observed cellular imaging using CLSM. As shown in Figure $5 a, c$ and Figure S5a,c, the nanoprobe displayed weak 
fluorescence in the cells which showed a few nanoprobes were internalized by the U87 and HeLa cells. To enhance the targeting ability of the nanoprobe, cRGD as a targeting ligand was linked to the periphery of the BSA-PCL conjugate coated QDs via carbodiimine chemistry, and the cellular internalization of cRGD-decorated nanoprobe was evaluated. From the images (Figure 5b,d and Figure S5b,d), we could see that the cRGDdecorated BSA-PCL conjugate coated QDs was significantly internalized and exhibited the strong fluorescence in two kinds of tumor cells. The flow cytometry results further verified these findings. The cell uptakes of the nanoprobe were enhanced after decoration with cRGD due to the high binding affinity of the peptide of cRGD with overexpressed integrin $\alpha_{\mathrm{v}} \beta_{3}$ located in cell membranes of U87 and HeLa cells. ${ }^{38-41}$ These cellular imaging data indicate BSA-PCL conjugate coated QDs has low nonspecific adsorption to the cells and the decorated nanoprobe with cRGD can actively target to the integrin $\alpha_{\mathrm{v}} \beta_{3}$-overexpressed cells.

3.4. In Vitro Cytotoxicity of the NIR Fluorescence Nanoprobe. We assessed the cytotoxicity of the pure amphiphilic BSA-PCL conjugate and the constructed CIS/ $\mathrm{ZnS}$ nanoprobe in both U87 and HeLa cells by the MTT assay to evaluate their biocompatibility. Figure 6 showed the viability
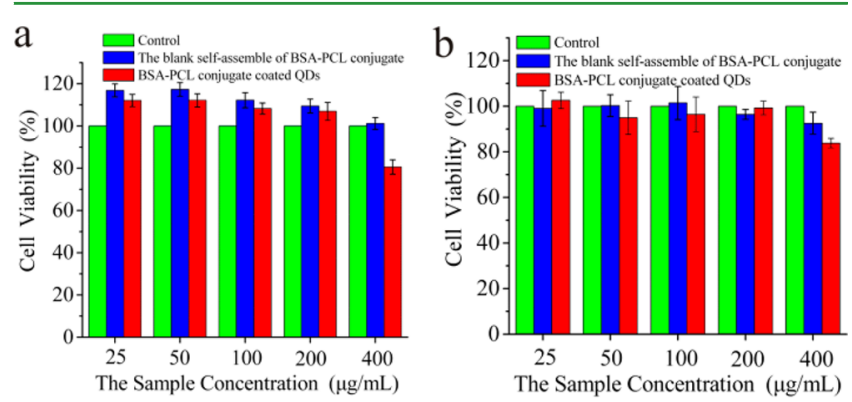

Figure 6. In vitro cell viability of U87 cells (a) and HeLa cells (b) incubated with the blank self-assemble of amphiphilic BSA-PCL conjugate and the NIR fluorescence nanoprobe for $24 \mathrm{~h}$ at $37^{\circ} \mathrm{C}$ with different concentrations: $25,50,100,200$, and $400 \mu \mathrm{g} / \mathrm{mL}$. The pure DMEM served as the control.

of cells after incubation with the blank self-assembly of the amphiphilic BSA-PCL conjugate and the NIR fluorescence nanoprobe for $24 \mathrm{~h}$. The results exhibited that no obvious cytotoxicity was observed for the pure amphiphilic BSA-PCL conjugate and the constructed $\mathrm{CIS} / \mathrm{ZnS}$ nanoprobe even with a concentration as high as $400 \mu \mathrm{g} / \mathrm{mL}$. The previous studies have demonstrated that surface modification of QDs is very important to reduce toxicity and in particular, coating with BSA could provide protection from photooxidation and improve the biocompatibility of QDs. ${ }^{15,42}$ In our study, the tailor-designed amphiphilic BSA-PCL conjugate has been proven to have good biocompatibility and a high degree of safety, ${ }^{21}$ and surface engineering of CIS/ZnS QDs with the prepared BSA-PCL conjugate imparts good biocompatibility to QDs. Building on the above-mentioned MTT results, it can be deduced that the constructed CIS/ZnS nanoprobe is highly biocompatible and nontoxic to the living cells.

3.5. In Vivo Imaging. Finally, we investigated the feasibility of the constructed NIR fluorescence nanoprobe for in vivo imaging. A volume of $200 \mu \mathrm{L}$ of the NIR fluorescence nanoprobe solution containing $100 \mu \mathrm{g}$ CIS/ZnS QDs were injected intravenously via the tail vein into a 5-week-old normal female nude mice and that in the control group underwent the same way of injection with physiological saline simultaneously. Subsequently, whole body images were carried out and analyzed at various time points though Maestro In-Vivo Imaging System. As shown in Figure S6a, only a little of the fluorescent signals were observed in liver at $10 \mathrm{~min}$ after intravenous injection. The fluorescent signals in liver gradually increased with time elapsing, and at $1 \mathrm{~h}$ post injection intense fluorescent signals were observed in the liver. In vivo imaging at 3 and 5 h clearly showed the fluorescent signals was enhanced in the spleen. As shown with in vivo imaging, the fluorescent signals were mainly observed in the liver and spleen due to the capture of QDs by the reticuloendothelial system (RES). The fluorescent signals were observed very little in the whole body in the control group. After the observation of $5 \mathrm{~h}$, the mice were sacrificed to investigate the ex vivo biodistribution. The major organs were collected for fluorescence imaging and ROI analysis with the assistance of imaging software, shown in Figure S6b,c. In accordance with the ex vivo imaging, the ROI analysis result further confirms the accumulation of QDs in liver and spleen. The results demonstrate the NIR fluorescence nanoprobe can be used for in vivo imaging, and our next step is to investigate the tumor targeting capability of in vivo imaging and supersession of the constructed nanoprobe in tumorbearing nude mice.

\section{CONCLUSIONS}

In conclusion, we have developed a simple, straightforward, and reproducible method for direct fabrication of the NIR fluorescence nanoprobe by coating CIS/ZnS QDs with a novel amphiphilic BSA-PCL bioconjugate for fluorescence imaging. This bioconjugate with a tailor-designed structure of BSA as the hydrophilic segment and PCL as the hydrophobic part was prepared by chemically coupling the hydrophobic maleimide-functionalized PCL chain to BSA via the maleimide-sulfhydryl reaction. The developed NIR fluorescence nanoprobe exhibited excellent fluorescent properties, good colloidal stability in PBS buffer $(\mathrm{pH}=7.4)$ and PBS buffer $(\mathrm{pH}$ $=7.4$ ) with $10 \%$ FBS, and high biocompatibility and nontoxicity to the living cells. More importantly, the constructed NIR fluorescence nanoprobe showed reduced nonspecific cellular imaging due to the protection of BSA which was on the outer surface of the nanoprobe, and the cRGDdecorated nanoprobe can be readily taken up by the tumor cells used for targeted tumor cells imaging. Finally, the feasibility of the NIR fluorescence nanoprobe is verified by in vivo imaging. This approach for the construction of the NIR fluorescence nanoprobe with a novel amphiphilic bioconjugate will find wide application in the preparation of multimodality imaging agents and versatile theranostics.

\section{ASSOCIATED CONTENT}

\section{Supporting Information}

The Supporting Information is available free of charge on the ACS Publications website at DOI: 10.1021/acsami.5b05406.

Additional characterization of the materials and nanoprobes $(\mathrm{PDF})$

\section{AUTHOR INFORMATION}

\section{Corresponding Authors}

*E-mail: jinchang@tju.edu.cn. Phone: +86-022-27401821. Fax: +86-022-27401821. 
*E-mail: tuyu@suda.edu.cn. Phone: +86-0512-65882615. Fax: +86-0512-65884830.

\section{Author Contributions}

${ }^{\perp}$ Z.L. and N.C. contributed equally to this work.

Notes

The authors declare no competing financial interest.

\section{ACKNOWLEDGMENTS}

The authors gratefully acknowledge National Natural Science Foundation of China (Grants 51373117, 51303126), Key Project of Tianjin Natural Science Foundation (Grant 13JCZDJC33200), Tianjin Research Program of Application Foundation and Advanced Technology (Grant 12JCZDJC26000), Doctoral Base Foundation of Educational Ministry of China (Grant 20120032110027), and Graduate Student Innovation Project of Jiangsu Province (Grant CXZZ13_0825).

\section{REFERENCES}

(1) Michalet, X.; Pinaud, F. F.; Bentolila, L. A.; Tsay, J. M.; Doose, S.; Li, J. J.; Sundaresan, G.; Wu, A. M.; Gambhir, S. S.; Weiss, S. Quantum Dots for Live Cells, In Vivo Imaging, and Diagnostics. Science 2005, 307, 538-544.

(2) Gu, L.; Hall, D. J.; Qin, Z.; Anglin, E.; Joo, J.; Mooney, D. J.; Howell, S. B.; Sailor, M. J. In Vivo Time-Gated Fluorescence Imaging with Biodegradable Luminescent Porous Silicon Nanoparticles. Nat. Commun. 2013, 4, 2326-2333.

(3) Yuan, L.; Lin, W.; Zheng, K.; He, L.; Huang, W. Far-Red to Near Infrared Analyte-Responsive Fluorescent Probes Based on Organic Fluorophore Platforms for Fluorescence Imaging. Chem. Soc. Rev. 2013, 42, 622-661.

(4) Yong, K.-T.; Roy, I.; Hu, R.; Ding, H.; Cai, H.; Zhu, J.; Zhang, X.; Bergey, E. J.; Prasad, P. N. Synthesis of Ternary $\mathrm{CuInS}_{2} / \mathrm{ZnS}$ Quantum Dot Bioconjugates and Their Applications for Targeted Cancer Bioimaging. Integr. Biol. 2010, 2, 121-129.

(5) Hilderbrand, S. A.; Weissleder, R. Near-Infrared Fluorescence: Application to In Vivo Molecular Imaging. Curr. Opin. Chem. Biol. 2010, 14, 71-79.

(6) Wang, R.; Zhang, F. NIR Luminescent Nanomaterials for Biomedical Imaging. J. Mater. Chem. B 2014, 2, 2422-2443.

(7) Pons, T.; Pic, E.; Lequeux, N.; Cassette, E.; Bezdetnaya, L.; Guillemin, F.; Marchal, F.; Dubertret, B. Cadmium-Free $\mathrm{CuInS}_{2} / \mathrm{ZnS}$ Quantum Dots for Sentinel Lymph Node Imaging with Reduced Toxicity. ACS Nano 2010, 4, 2531-2538.

(8) Deng, D.; Chen, Y.; Cao, J.; Tian, J.; Qian, Z.; Achilefu, S.; Gu, Y. High-Quality $\mathrm{CuInS}_{2} / \mathrm{ZnS}$ Quantum Dots for In Vitro and In Vivo Bioimaging. Chem. Mater. 2012, 24, 3029-3037.

(9) Foda, M. F.; Huang, L.; Shao, F.; Han, H. Biocompatible and Highly-Luminescent Near Infrared $\mathrm{CuInS}_{2} / \mathrm{ZnS}$ Quantum Dots Embedded Silica Beads for Cancer Cell Imaging. ACS Appl. Mater. Interfaces 2014, 6, 2011-2017.

(10) Li, L.; Daou, T. J.; Texier, I.; Kim Chi, T. T.; Liem, N. Q.; Reiss, P. Highly Luminescent $\mathrm{CuInS}_{2} / \mathrm{ZnS}$ Core/Shell Nanocrystals: Cadmium-Free Quantum Dots for In Vivo Imaging. Chem. Mater. 2009, 21, 2422-2429.

(11) Wang, M.; Liu, X.; Cao, C.; Wang, L. Highly Luminescent $\mathrm{CuInS}_{2}-\mathrm{ZnS}$ Nanocrystals: Achieving Phase Transfer and Nuclear Homing Property Simultaneously Through Simple TTAB Modification. J. Mater. Chem. 2012, 22, 21979-21986.

(12) Cheng, C.-Y.; Ou, K.-L.; Huang, W.-T.; Chen, J.-K.; Chang, J.Y.; Yang, C.-H. Gadolinium-Based $\mathrm{CuInS}_{2} / \mathrm{ZnS}$ Nanoprobe for DualModality Magnetic Resonance/Optical Imaging. ACS Appl. Mater. Interfaces 2013, 5, 4389-4400.

(13) Biju, V.; Itoh, T.; Ishikawa, M. Delivering Quantum Dots to Cells: Bioconjugated Quantum Dots for Targeted and Nonspecific
Extracellular and Intracellular Imaging. Chem. Soc. Rev. 2010, 39, 3031-3056.

(14) Jiang, S.; Win, K. Y.; Liu, S.; Teng, C. P.; Zheng, Y.; Han, M.-Y. Surface-Functionalized Nanoparticles for Biosensing and ImagingGuided Therapeutics. Nanoscale 2013, 5, 3127-3148.

(15) Sun, H.; Zhang, F.; Wei, H.; Yang, B. The Effects of Composition and Surface Chemistry on the Toxicity of Quantum Dots. J. Mater. Chem. B 2013, 1, 6485-6494.

(16) Jańczewski, D.; Tomczak, N.; Han, M.-Y.; Vancso, G. J. Synthesis of Functionalized Amphiphilic Polymers for Coating Quantum Dots. Nat. Protoc. 2011, 6, 1546-1553.

(17) Tomczak, N.; Liu, R; Vancso, J. G. Polymer-Coated Quantum Dots. Nanoscale 2013, 5, 12018-12032.

(18) Lin, C. A. J.; Sperling, R. A.; Li, J. K.; Yang, T. Y.; Li, P. Y.; Zanella, M.; Chang, W. H.; Parak, W. J. Design of an Amphiphilic Polymer for Nanoparticle Coating and Functionalization. Small 2008, 4, 334-341.

(19) Zhang, B.; Wang, X.; Liu, F.; Cheng, Y.; Shi, D. Effective Reduction of Nonspecific Binding by Surface Engineering of Quantum Dots with Bovine Serum Albumin for Cell-Targeted Imaging. Langmuir 2012, 28, 16605-16613.

(20) Xia, B.; Zhang, W.; Shi, J.; Xiao, S.-j. Engineered Stealth Porous Silicon Nanoparticles via Surface Encapsulation of Bovine Serum Albumin for Prolonging Blood Circulation. ACS Appl. Mater. Interfaces 2013, 5, 11718-11724.

(21) Liu, S.; Shi, F.; Chen, L.; Su, X. Bovine Serum Albumin Coated $\mathrm{CuInS}_{2}$ Quantum Dots as a Near-Infrared Fluorescence Probe for 2, 4, 6-Trinitrophenol Detection. Talanta 2013, 116, 870-875.

(22) Nakane, Y.; Sasaki, A.; Kinjo, M.; Jin, T. Bovine Serum Albumin-Coated Quantum Dots as a Cytoplasmic Viscosity Probe in a Single Living Cell. Anal. Methods 2012, 4, 1903-1905.

(23) Liu, Z.; Dong, C.; Wang, X.; Wang, H.; Li, W.; Tan, J.; Chang, J. Self-Assembled Biodegradable Protein-polymer Vesicle as Tumortargeted Nanocarrier. ACS Appl. Mater. Interfaces 2014, 6, 2393-2400.

(24) Guo, W.; Chen, N.; Tu, Yu; Dong, C.; Zhang, B.; Hu, C.; Chang, J. Synthesis of Zn-Cu-In-S/ZnS Core/Shell Quantum Dots with Inhibited Blue-Shift Photoluminescence and Applications for Tumor Targeted Bioimaging. Theranostics 2013, 3, 99-108.

(25) Wu, Y.; Chakrabortty, S.; Gropeanu, R. A.; Wilhelmi, J.; Xu, Y.; Er, K. S.; Kuan, S. L.; Koynov, K.; Chan, Y.; Weil, T. pH-Responsive Quantum Dots via an Albumin Polymer Surface Coating. J. Am. Chem. Soc. 2010, 132, 5012-5014.

(26) Eisele, K.; Gropeanu, R.; Musante, A.; Glasser, G.; Li, C.; Muellen, K.; Weil, T. Tailored Albumin-Based Copolymers for Receptor-Mediated Delivery of Perylenediimide Guest Molecules. Macromol. Rapid Commun. 2010, 31, 1501-1508.

(27) Elzoghby, A. O.; Samy, W. M.; Elgindy, N. A. Albumin-Based Nanoparticles as Potential Controlled Release Drug Delivery Systems. J. Controlled Release 2012, 157, 168-182.

(28) Quan, Q.; Xie, J.; Gao, H.; Yang, M.; Zhang, F.; Liu, G.; Lin, X.; Wang, A.; Eden, H. S.; Lee, S.; Zhang, G.; Chen, X. HSA Coated Iron Oxide Nanoparticles as Drug Delivery Vehicles for Cancer Therapy. Mol. Pharmaceutics 2011, 8, 1669-1676.

(29) Wu, Y.; Eisele, K.; Doroshenko, M.; Algara-Siller, G.; Kaiser, U.; Koynov, K.; Weil, T. A Quantum Dot Photoswitch for DNA Detection, Gene Transfection, and Live-Cell Imaging. Small 2012, 8, 3465-3475.

(30) Gagner, J. E.; Kim, W.; Chaikof, E. L. Designing Protein-Based Biomaterials for Medical Applications. Acta Biomater. 2014, 10, 15421557.

(31) Guo, S.; Qiao, Y.; Wang, W.; He, H.; Deng, L.; Xing, J.; Xu, J.; Liang, X.-J.; Dong, A. Poly ( $\varepsilon$-caprolactone)-graft-Poly (2-(N, Ndimethylamino) ethyl methacrylate) Nanoparticles: $\mathrm{pH}$ Dependent Thermo-Sensitive Multifunctional Carriers for Gene and Drug Delivery. J. Mater. Chem. 2010, 20, 6935-6941.

(32) Wu, P.; Yan, X.-P. Doped Quantum Dots for Chemo/ Biosensing and Bioimaging. Chem. Soc. Rev. 2013, 42, 5489-5521.

(33) Muro, E.; Pons, T.; Lequeux, N.; Fragola, A.; Sanson, N.; Lenkei, Z.; Dubertret, B. Small and Stable Sulfobetaine Zwitterionic 
Quantum Dots for Functional Live-Cell Imaging. J. Am. Chem. Soc. 2010, 132, 4556-4557.

(34) Breus, V. V.; Heyes, C. D.; Tron, K.; Nienhaus, G. U. Zwitterionic Biocompatible Quantum Dots for Wide pH Stability and Weak Nonspecific Binding to Cells. ACS Nano 2009, 3, 2573-2580.

(35) Zhou, Z.; Shen, Y.; Tang, J.; Fan, M.; Van Kirk, E. A.; Murdoch, W. J.; Radosz, M. Charge-Reversal Drug Conjugate for Targeted Cancer Cell Nuclear Drug Delivery. Adv. Funct. Mater. 2009, 19, 3580-3589.

(36) Liu, T.; Li, X.; Qian, Y.; Hu, X.; Liu, S. Multifunctional pHDisintegrable Micellar Nanoparticles of Asymmetrically Functionalized $\beta$-Cyclodextrin-Based Star Copolymer Covalently Conjugated with Doxorubicin and DOTA-Gd Moieties. Biomaterials 2012, 33, 25212531.

(37) Koo, H.; Huh, M. S.; Ryu, J. H.; Lee, D.-E.; Sun, I.-C.; Choi, K.; Kim, K.; Kwon, I. C. Nanoprobes for Biomedical Imaging in Living Systems. Nano Today 2011, 6, 204-220.

(38) Choi, H. S.; Liu, W.; Liu, F.; Nasr, K.; Misra, P.; Bawendi, M. G.; Frangioni, J. V. Design Considerations for Tumour-Targeted Nanoparticles. Nat. Nanotechnol. 2010, 5, 42-47.

(39) Cai, W.; Chen, X. Preparation of Peptide-Conjugated Quantum Dots for Tumor Vasculature-Targeted Imaging. Nat. Protoc. 2008, 3, 89-96.

(40) Smith, A. M.; Duan, H.; Mohs, A. M.; Nie, S. Bioconjugated Quantum Dots for In Vivo Molecular and Cellular Imaging. Adv. Drug Delivery Rev. 2008, 60, 1226-1240.

(41) Mulder, W. J.; Koole, R.; Brandwijk, R. J.; Storm, G.; Chin, P. T.; Strijkers, G. J.; de Mello Donegá, C.; Nicolay, K.; Griffioen, A. W. Quantum Dots with a Paramagnetic Coating as a Bimodal Molecular Imaging Probe. Nano Lett. 2006, 6, 1-6.

(42) Hoshino, A.; Hanada, S.; Yamamoto, K. Toxicity of Nanocrystal Quantum Dots: the Relevance of Surface Modifications. Arch. Toxicol. 2011, 85, 707-720.

\section{NOTE ADDED AFTER ASAP PUBLICATION}

This paper was published ASAP on August 18, 2015, with errors to the SI. The updated SI has been uploaded and reposted on August 24, 2015. 